\title{
Investigation of the effect of the quality of inspection techniques on the optimal inspection interval for structures
}

\author{
E. Sheils ${ }^{\mathrm{a}}$, A. O'Connor ${ }^{\mathrm{a} *}$, F. Schoefs ${ }^{\mathrm{b}}$ and D. Breysse ${ }^{\mathrm{c}}$ \\ ${ }^{a}$ Department of Civil Engineering, Trinity College Dublin, Dublin 2, Ireland; ${ }^{b}$ Institute of Civil and Mechanical Research (GeM), \\ UMR-CNRS 6183, Nantes Atlantic University, 2 rue de la Houssiniere, B.P. 9220844322 Nantes Cedex 03, France; 'GHYMAC, \\ University Bordeaux 1, bât 18, Avenue des facultés, F-33405 Talence cedex, France
}

\begin{abstract}
Quantitative data from inspections are necessary to determine the state of a deteriorating structure and to identify the optimal maintenance strategy for the remaining life of the structure. This article presents the development of a two-stage inspection based maintenance management framework, which provides the owner/manager of a structure with a decision tool to optimise the service life costs of a structure. The two stages of an inspection are considered here, detection of defects present and sizing of detected defects. Using this framework, the optimal inspection interval for this study, based on the minimisation of the service life costs, is determined and the effect of inspection quality of the two techniques is studied. By modelling the detection and sizing stage of an inspection separately, the optimum combination of techniques can be determined.
\end{abstract}

Keywords: detection; inspection; maintenance; Markov; sizing

\section{Introduction}

In the US about 5000 bridges become classified as deficient each year and the estimated cost of repair and rehabilitation of these deteriorating structures has been estimated at $\$ 1.3$ trillion (Enright and Frangopol 1999). Therefore, there has been a lot of research carried out over the last decade to develop methods of maintenance management which optimise maintenance budgets over the service life of a structure, yet still ensure that the safety of the structure is not compromised (Estes and Frangopol 1999, Radojicic et al. 2001, Stewart 2001, Faber and Sorensen 2002, Kong and Frangopol 2004, 2005, Stewart et al. 2004, O'Connor and O'Brien 2005, O'Connor and Eichinger 2007, O'Connor and Enevoldsen 2009). Since many of these methods rely on quantitative data from inspections, rather than qualitative and subjective data, the inspection and monitoring of structures is a vital step in this process to provide ongoing information on the current state and deterioration of these structures.

The focus of this article is on the development of an inspection-based maintenance management framework, which considers the two stages of an inspection, the detection of a defect and the sizing assessment of a detected defect. In many previous studies, only the detection stage of an inspection was considered, where the quality of the inspection method was modelled using various parameters, such as probability of detection (PoD) and probability of false alarm (PFA) (Rouhan and Schoefs 2003), PoD and probability of false indications (Straub and Faber 2003), PoD and false call probability (Zhang and Mahadevan 2001, Chung et al. 2006).

In this study, by considering the interaction of the two stages of an inspection, the optimum combination of techniques for both inspections can be determined. Since each stage of an inspection is carried out for a distinct purpose, different parameters are used to represent each procedure, enabling the owner/manager of a structure to choose the most suitable inspection technique for each stage of the inspection.

Firstly, an inspection is carried out to detect defects present in the structure. In this study, for a particular Non Destructive Testing (NDT) tool used to carry out an inspection, the PoD and the PFA are used to indicate the quality of the inspection method for detection. Subsequently, the sizing assessment inspection is carried out to size defects that are detected by the first inspection. For this analysis, two new parameters are introduced, the probability of good assessment (PGA) and probability of wrong assessment (PWA).

Using this framework, the optimal inspection interval for this study, based on the minimisation of the service life costs, is determined and the effect of inspection quality of the two techniques is studied. By modelling the detection and sizing stage of an

\footnotetext{
*Corresponding author. Email: alan.oconnor@tcd.ie
} 
inspection separately, the optimum combination of techniques can be determined.

\section{Theoretical background}

Due to the inherent uncertainty associated with inspection results, many of the variables which are used to simulate inspection outcomes are modelled stochastically. Given the size of the defect, and the inspection method being used, there is a certain PoD (Madsen et al. 1987, Faber and Sorensen 2002, Onoufriou and Frangopol 2002). Therefore, in this case, inspection results for detection and sizing assessment are modelled using probabilistic methods which take these uncertainties into account.

\section{Probabilistic modelling of inspection results - detection}

In this study, it is assumed that inspections are carried out on structures every $\Delta T$ years. The PoD and the PFA are used to indicate the quality of an inspection method for detection. The PoD is the probability that a defect is detected by the inspection, given that a defect is present, Equation (1), and the PFA is the probability that a defect is detected by the inspection, given that no defect greater than the detection threshold is present, Equation (2).

$$
\begin{aligned}
& \mathrm{PoD}=P\left(\hat{d}_{1} \geq d_{\min } \mid d \geq d_{\min }\right) \\
& \mathrm{PFA}=P\left(\hat{d}_{1} \geq d_{\min } \mid d<d_{\min }\right)
\end{aligned}
$$

where $\hat{d}_{1}=$ size of the detected defect (from inspection 1), $d=$ actual size of the defect and $d_{\min }=$ detection threshold. The PoD depends on the size of the defect, the detection threshold and the noise, whereas, PFA depends only on the detection threshold and the noise. Figure 1 illustrates that the $\mathrm{PoD}$ is the probability that the 'signal+noise' is greater than the detection threshold, and the PFA is the probability that the 'noise' is greater than the detection threshold (Rouhan and Schoefs 2003). The 'noise' distribution represents the error due to environmental conditions, human interference and the nature of what is being measured.

\section{Probabilistic modelling of inspection results - sizing assessment}

A second inspection is then carried out to size defects that are detected by the first inspection. For this analysis, two new parameters are introduced, the PGA and PWA. In addition, a distinction is made between good and wrong assessments which lead to repair $\left(\mathrm{PGA}_{\mathrm{R}}, \mathrm{PWA}_{\mathrm{R}}\right)$, and those which lead to no repair $\left(\mathrm{PGA}_{\mathrm{NR}}, \mathrm{PWA}_{\mathrm{NR}}\right)$, Equations (3)-(8). It is assumed

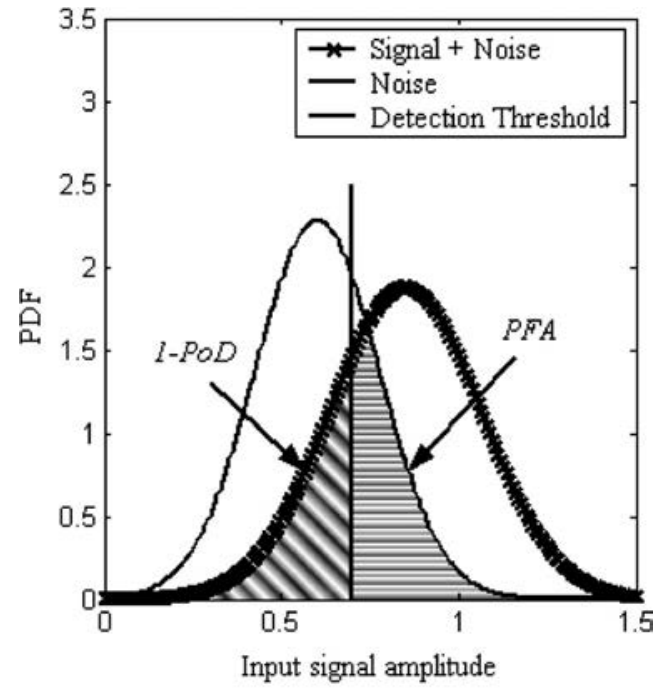

N Probability of a missed detection

Probability of a false alarm

Figure 1. The effect of noise on inspection results (for a distribution of defects).

that any defects which are sized and found to be greater than the critical defect size, $d_{c}$, (which can be specified by the owner/manager of the structure) are repaired.

$$
\begin{gathered}
\mathrm{PGA}_{\mathrm{R}}=P\left(\hat{d}_{2} \geq d_{c} \mid d \geq d_{c} \text { and } \hat{d}_{1} \geq d_{\mathrm{min}}\right) \\
\mathrm{PGA}_{\mathrm{NR}}=P\left(\hat{d}_{2}<d_{c} \mid d<d_{c} \text { and } \hat{d}_{1} \geq d_{\mathrm{min}}\right) \\
\mathrm{PWA}_{\mathrm{R}}=P\left(\hat{d}_{2} \geq d_{c} \mid d<d_{c} \text { and } \hat{d}_{1} \geq d_{\min }\right) \\
\mathrm{PWA}_{\mathrm{NR}}=P\left(\hat{d}_{2}<d_{c} \mid d \geq d_{c} \text { and } \hat{d}_{1} \geq d_{\min }\right)
\end{gathered}
$$

Note:

$$
\begin{aligned}
& \mathrm{PGA}_{\mathrm{NR}}=1-\mathrm{PWA}_{\mathrm{R}} \\
& \mathrm{PWA}_{\mathrm{NR}}=1-\mathrm{PGA}_{\mathrm{R}}
\end{aligned}
$$

where $\hat{d}_{2}=$ size of the defect from inspection 2, $d=$ actual size of the defect and $d_{c}=$ critical defect size. In this case, for a given inspection, both the PGA and PWA depend on the defect size, the critical defect size (threshold) and the noise.

Therefore, the inspection can be modelled using just one distribution, as shown in Figure 2, where $\bar{d}_{\mathrm{i}}$ is the mean defect size within a group $i$. The $\mathrm{PGA}_{\mathrm{R}}$ is the probability that the 'signal + noise' is greater than the critical defect size (leading to repair), given that the actual defect is greater than the critical defect 
size $d_{c}$, (Figure 2a), and the $\mathrm{PGA}_{\mathrm{NR}}$ is the probability that the 'signal + noise' is less than the critical defect size (leading to no repair), given that the defect is less than the critical defect size, (Figure 2b). Similarly, the PWA $_{R}$ is the probability that the 'signal + noise' is greater than the critical defect size (leading to repair), given that the actual defect is less than the critical defect size, (Figure 2b), and the $\mathrm{PWA}_{\mathrm{NR}}$ is the probability that the 'signal + noise' is less than the critical defect size (leading to no repair), given that the defect is greater than the critical defect size (Figure 2a).

\section{Development of a maintenance management model}

For this study, the total range of defect sizes is broken into 10 defect groups and a record is kept of the number of defects in each of these groups each year, assuming a total of 100 defects. Assuming that the state of the structure in each time period depends only on the state of the structure and the action applied to it in the preceding period (Ang and Tang 1975), a Markov process is employed to simulate the growth/ evolving deterioration and repair of a population of defects over time (Scherer and Glagola 1994, Roelfstra et al. 2004). In practice, many Bridge Management Systems use Markov chains to predict future deterioration and repair (Czepiel 1995, Frangopol et al. 2001, Adey et al. 2003, Bakht and Mutsuyoshi 2005, Rens et al. 2005). Although the condition of the structure is recorded using discrete states and at discrete times using a Markovian-based methodology (unlike a reliability based approach), the physical process of deterioration of a structure is relatively stable, and inspections and maintenance are carried out at discrete times, therefore, the state of a structure is only required at these discrete times when decisions are made
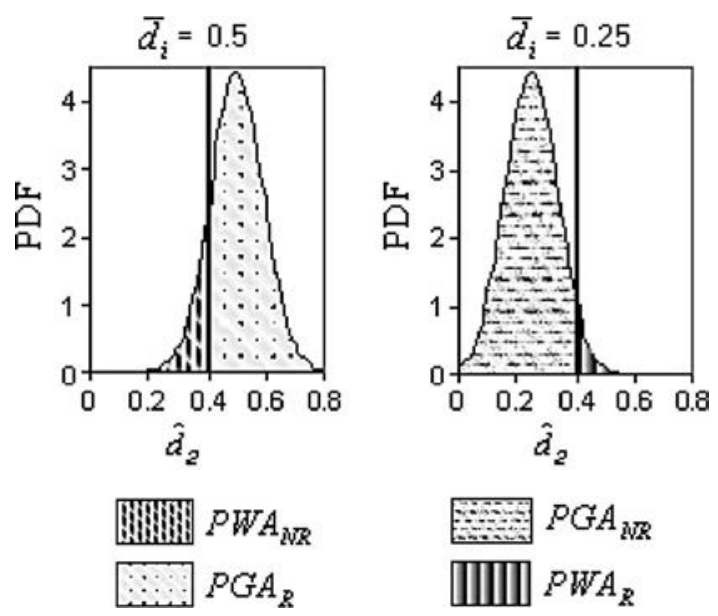

Figure 2. Example of the effect of noise on sizing inspection results $\left(d_{c}=0.4\right)$.
(Corotis et al. 2005). Although a Markov-based approach is the chosen method of simulation in this study, it is recognised that the deterioration, maintenance and repair of a structure could also be simulated using a reliability-based approach, or using a combination of both methods (Estes and Frangopol 2001).

Two Markov matrices $(\mathbf{N} \times \mathbf{N}$, where $\mathbf{N}$ is the number of defect groups) are developed to simulate the evolution of a population of defects over time, one simulates the growth and failure of the defects between inspections and the other simulates the growth, repair and failure of the defects at the year of an inspection.

\section{Markov matrices}

When considering a Markov process, what is of interest is to calculate the probability that a defect moves from one group to a larger group. This probability is defined as $\mathbf{P}_{\mathbf{i j}}$, where $\mathbf{P}_{\mathbf{i j}}$ is the probability that a defect will move from group $i$ to group $j$ in one time step (Ang and Tang 1984, Cesare et al. 1992). Also, in the transition matrix $\mathbf{P}_{\mathbf{i j}}$ is the entry in the $i$ th row and the $j$ th column. The objective here is to create two Markov transition matrices using the specified parameters for growth, inspection, repair and failure. It is assumed that the defects return to the smallest defect group when repair/failure occurs. Therefore, at an inspection year, the first column in the matrix is controlled by the probability of repair and the probability of failure given that no repair is carried out, whereas, between inspections, this column is controlled by the probability of failure alone (Sheils et al. 2010).

The upper triangular part of the Markov matrix is controlled by the growth characteristics of the defects. To simulate the growth of the defects, two parameters are defined. The first is $\alpha$, which describes the growth rate of a defect and therefore controls how quickly a defect moves from one defect group to another defect group. The other parameter is $g$, which determines how gradual or sudden the growth of an individual defect is. This parameter controls whether defects develop gradually and just move from one defect group to the next, or whether the growth of a defect is more abrupt, causing it to move from one group to a defect group a few sizes larger (rather than the one next to it). This allows many different forms of deterioration mechanism, which are associated with different environments and materials, to be simulated using this approach. Depending on the limit state being considered, the owner/manager of a structure will be concerned with different forms of deterioration (e.g. in relation to Serviceability Limit State (SLS), the owner/manage may be concerned with crack growth due to reinforced concrete corrosion in concrete structures), and based 
on field data or experimental results in the laboratory, these parameters can be estimated to predict the deterioration of a structure over time. A higher growth rate means that there is a lower probability that the defect will remain in the same defect group after each time step, and a lower growth rate increases the likelihood that it will stay in the same group after the same period of time. For example, crack growth in a reinforced concrete structure in an aggressive marine environment is more likely to develop at a faster rate than a structure inland where the exposure to chlorides is minimal, and will therefore be more likely to move to a larger defect group within a certain time interval.

To model this behaviour, the diagonal of the transition matrix is controlled by $(1-\alpha)$ alone, for $(0<\alpha<1)$. Each row in the transition matrix must sum to 1.0. Therefore, the remaining portion of the probabilities $(\alpha)$ must be distributed between the other groups (or the cells in that row of the matrix). The ratio by which they are divided is controlled by $g$, as $g$ is used to describe the growth kinetics of the defects. For a high value of $g$, (e.g. $g=5$ ), the growth of an individual defect is modelled as smooth/gradual (Figure 3).

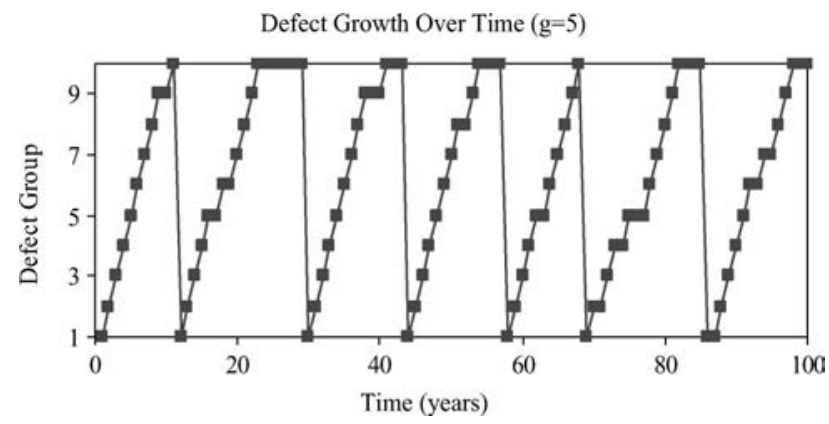

Figure 3. Gradual defect growth of an individual defect $(g=5)$.
This form of growth would correspond to a deterioration process such as carbonation or chloride-induced corrosion in reinforced concrete as these processes develop gradually over time. In this case, the defect stays in the same group or moves to the next defect group, and does not readily skip groups by growing very suddenly in one time step (i.e. $\mathbf{P}_{\mathbf{i}, \mathbf{i}+\mathbf{1}} \approx \alpha$, and $\mathbf{P}_{\mathbf{i j}} \approx 0$ (for $\left.j>i+1\right)$ ). An example of the transition matrix describing this behaviour is shown in Figure 4. Lower values of $g$ used in the model describe more abrupt growth, as illustrated in Figure 5, with $\mathbf{P}_{\mathbf{i j}}$ (for $j>i$ ) more evenly spread, increasing the likelihood that a defect jumps straight from the group it is in to a much larger defect group, skipping the groups adjacent to it, as illustrated in the transition matrix presented in Figure 6. This allows many forms of deterioration mechanism to be simulated using the developed methodology.

To calculate the values of the entries in the upper triangular growth matrix ( $\mathbf{P}_{\mathbf{i j} \_ \text {GROWTH }}$ ) using $\alpha$ and $g$, another matrix $\mathbf{G}$ is created, Equation (9). This is an inverse power equation which is used to calculate the values in the upper triangular portion (growth part) of the matrix. This model was chosen as it allows values of $g=0$ and negative values of $g$ to be modelled, resulting in an increased versatility to simulate different forms of deterioration mechanism.

$$
\mathbf{G}_{\mathrm{ij}}=\left\{\begin{array}{cc}
\frac{1}{(j-i)^{g}} & \text { for } \quad j>i \\
0 & \text { for } j \leq i
\end{array}\right.
$$

From this matrix, the values of the transition matrix are calculated using Equation (10),

$$
\mathbf{P}_{\mathrm{ij} \_ \text {GROWTH }}=\frac{(\alpha)\left(\mathbf{G}_{\mathbf{i j}}\right)}{\sum \mathbf{G}_{\mathbf{i}}} \quad \text { for } j>i
$$

To simulate failure between inspections, the annual probability of failure for each group is calculated using

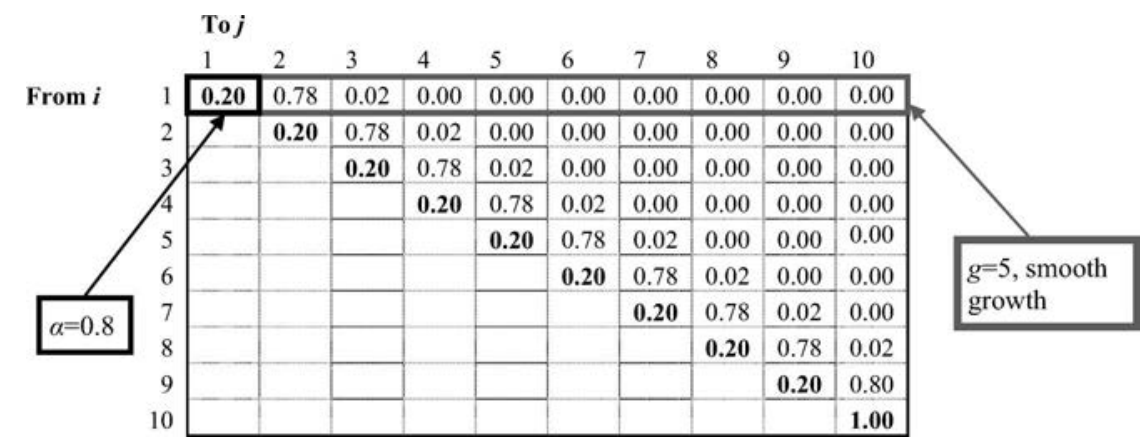

Figure 4. An example of a Markov matrix to simulate defect growth $(\alpha=0.8, g=5, N=10)$. 
the Weibull cumulative distribution function (Weibull 1951, Ang and Tang 1984) based on the mean size of the defects in each group, $\bar{d}_{i}$, Equation (11),

$$
p_{f}\left(\bar{d}_{i}\right)=1-\left[\exp -\left(\frac{\bar{d}_{i}-d_{1}}{d_{\text {ref_pf }}}\right)^{m}\right]
$$

where $d_{1}=$ limit defect size, $\mathrm{d}_{\text {ref_pf }}=$ reference defect size for the probability of failure and $m=$ Weibull exponent which determines the spread of the curve. The probability of failure for each group is then used to calculate the values for the first column in the Markov matrix, which simulates the behaviour of a population of defects between inspections.

At an inspection year, the first inspection is carried out to detect defects. If defects are detected, then a sizing assessment inspection is carried out, and repair is then carried out if the inspection indicates that the size of the defect is larger than the critical defect size. Due to errors and uncertainties associated with inspections, defects present in the structure may not be detected by the first inspection, and detected defects that are larger than the critical defect size may be sized incorrectly by the second inspection and therefore, may not be repaired. Both cases can lead to failures in

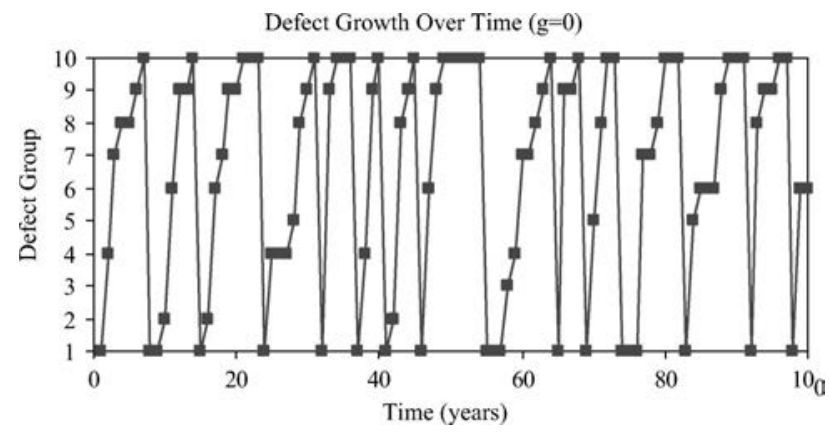

Figure 5. Abrupt defect growth of an individual defect $(g=0)$. the structure at an inspection year, and these failures are also simulated in the model. This process is illustrated in Figure 7. Therefore, at an inspection year, the first column of the Markov matrix is calculated using a combination of the probability of repair, the probability of failure given that no detection has occurred, and the probability of failure given that no repair has been carried out.

In this study, it is assumed that a defect returns to the smallest defect group following the repair of the defect (i.e. perfect repair). The probability of repair for each group is calculated analytically using the inspection parameters and the properties of the groups representing the range of defect sizes. The PoD/PFA and PGA/PWA are estimated for each group, given the mean and standard deviation of the defects in each group, the quality of the inspection method for detection $Q_{1}$ and sizing $Q_{2}$, and the mean value of the noise distribution, $n_{\text {mean }}$, which depends on environmental conditions and human interference. It is assumed for detection and sizing that the quality (and hence cost) of the inspection method is related to the distribution of the noise, $\sigma_{N D}$ (detection), Equation (12), $\sigma_{N A}$ (sizing assessment), Equation (13).

$$
\begin{aligned}
& \frac{\sigma_{N D}}{d_{\text {ref }}}=\frac{1}{Q_{1}} \\
& \frac{\sigma_{N A}}{d_{\text {ref }}}=\frac{1}{Q_{2}}
\end{aligned}
$$

where $d_{\text {ref }}=$ reference defect size.

When the first inspection is carried out to detect a defect, there can be two decision outcomes. One is to carry out a further assessment, and the other is to do nothing. Similarly, when a second inspection is carried out to assess the size of a defect, there can also be two decision outcomes. One is to repair, in which case the defect returns to the initial defect group, and the other is to carry out no repair. If at the detection stage no

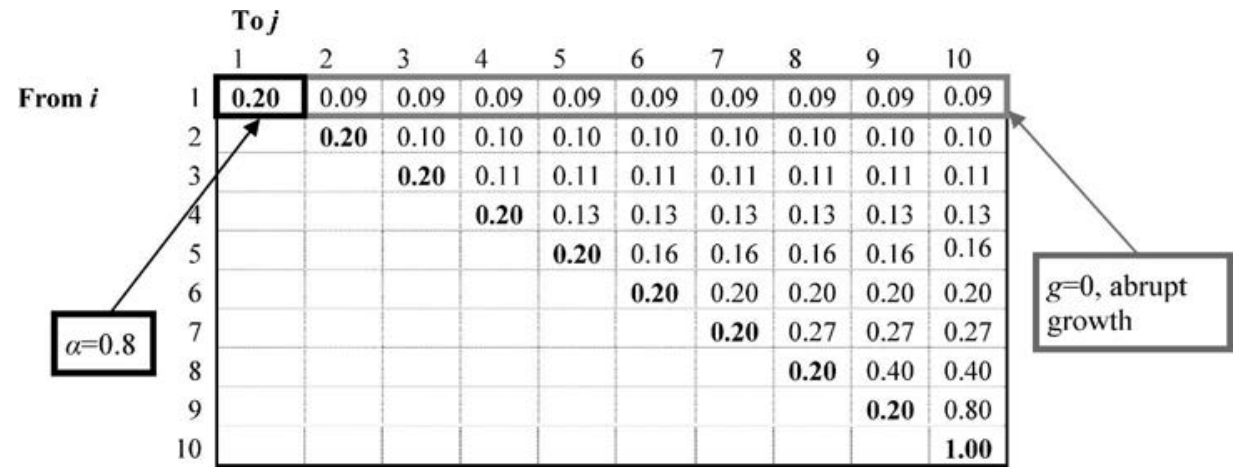

Figure 6. An example of a Markov matrix to simulate defect growth $(\alpha=0.8, g=0, N=10)$. 
further assessment is carried out, or if at the assessment stage no repair is carried out, there is still a remaining probability of failure. Similar to the event of repair, if failure occurs the defect is modelled as returning to the initial defect group, as illustrated in Figure 8 . The probability of these events is calculated analytically, and is used to determine the values to be inputted into the first column of the Markov matrix, which represents the probability that defects will return to the smallest group after failure or repair throughout the year.

Therefore, at an inspection year, the probability of repair or failure (event combination $\mathrm{R} \cup \mathrm{F}$ ) for each group, $\mathbf{P}_{\mathbf{R} \cup \mathbf{F}_{-} \text {, }}$, can be calculated using Equation (14),

$$
\begin{aligned}
\mathbf{p}_{\mathbf{R} \cup \mathbf{F} \mathbf{i}}= & \mathbf{P}_{\mathbf{i}}(\text { Repair })+\mathbf{P}_{\mathbf{i}}(\text { Failure due to No assessment }) \\
& +\mathbf{P}_{\mathbf{i}}(\text { Failure due to No repair })
\end{aligned}
$$

Once the Markov growth matrix for deterioration is developed and the values for the probability of repair/ failure at an inspection year and the values for the probability of failure between inspections are calculated for each group, they can be merged to develop two complete Markov matrices which can then be used to simulate the growth, inspection, repair and failure of a population of defects over time.

In this case, each defect group is assumed to have an initial population of defects, with the total number of defects summing to 100 . Using this methodology, the number of defects in each group is calculated on a yearly basis using the relevant Markov matrix, and the number of defects in each group from the previous year. The model is run until the number of defects in each group reaches a steady state. The stabilised number of defects in each group is then used to calculate the expected annual total cost of the structure.

\section{Cost analysis}

In the proposed methodology the expected annual cost of inspections $\left(E\left(C_{\mathrm{I}_{-} \text {tотаL }}\right)\right)$, repair $\left(E\left(C_{\mathrm{R}_{-} \text {tOTAL }}\right)\right)$ and failure $\left(E\left(C_{\mathrm{F}_{-} \text {TOTAL }}\right)\right)$ are considered, which are

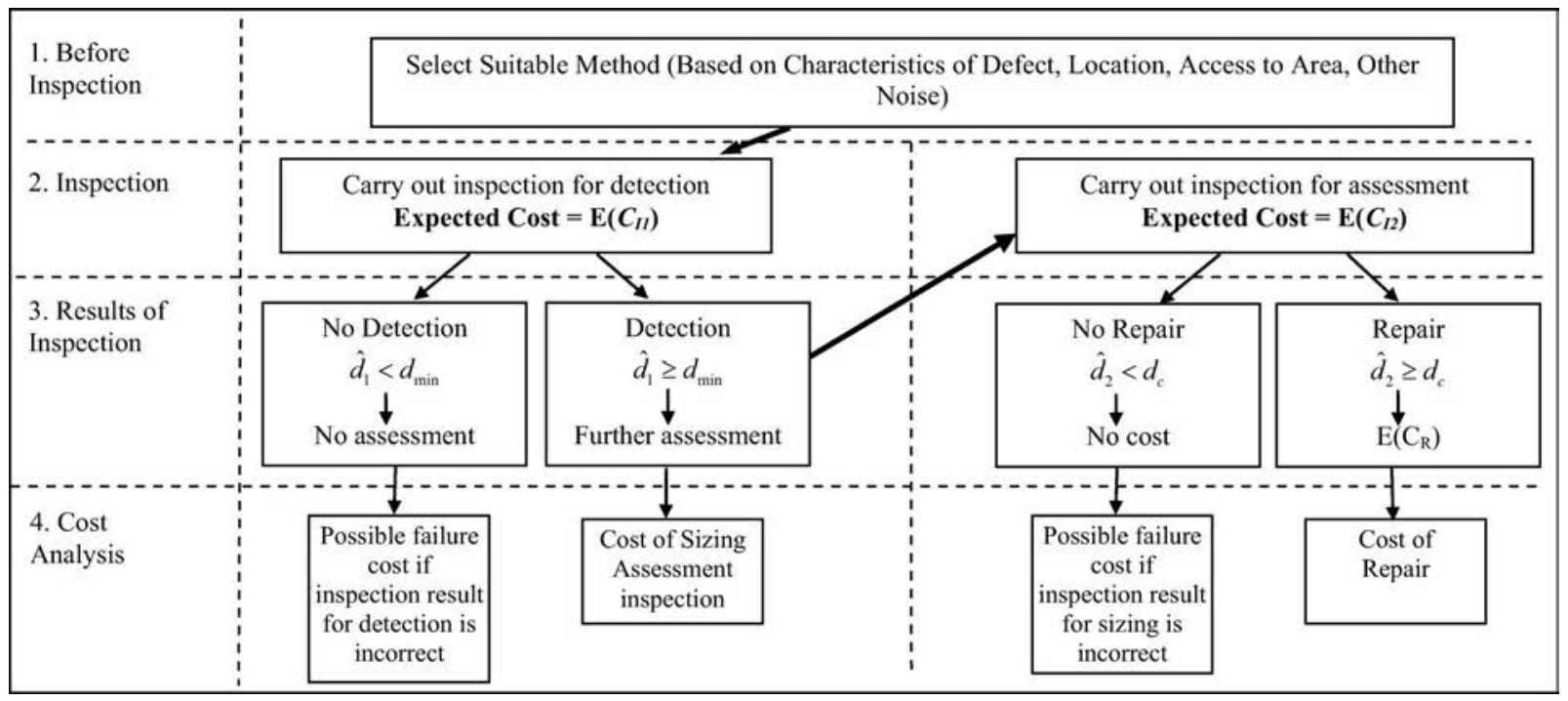

Figure 7. Inspection outcomes for a defect group.

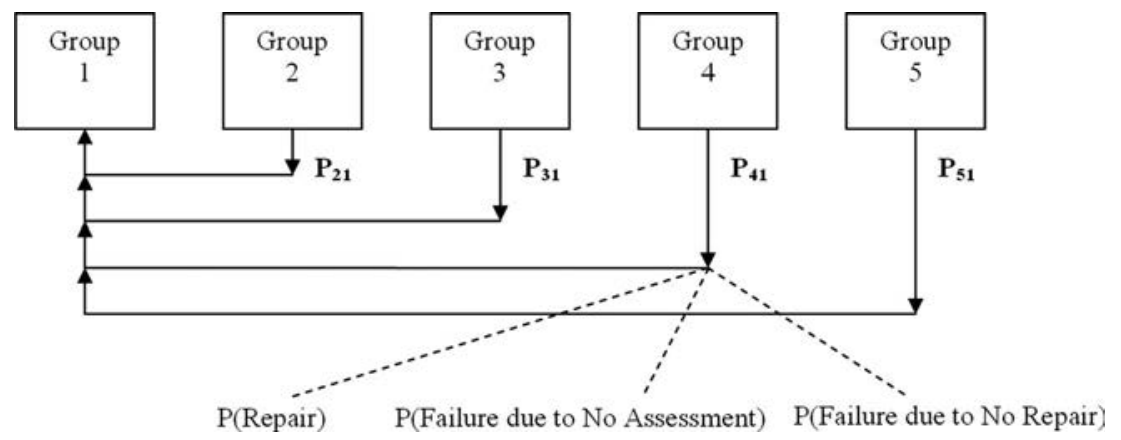

Figure 8. Schematic of repair/failure process for Markov chain (considering a perfect repair). 
summed to find the expected annual total cost of the structure $\left(E\left(C_{\text {TOTAL }}\right)\right)$. These are the direct costs associated with maintenance management of a structure or network of structures. It is recognised that other indirect costs, such as user delay costs and penalty costs for reduced serviceability could also be included, but since no data are available to accurately quantify the parameters associated with the assumed cost models, it was decided to model only the direct costs. The cost analysis presented in this article is used to compare the relative implications of different management decisions (e.g. different inspection quality, inspection intervals, etc.), and it is recognised that these cost models are subjective and should only be used to provide an indication of the relative benefits of different management strategies. Further work is required to develop accurate costing models for both direct and indirect costs. Although discounting is not considered as part of this study for simplicity, it is recognised that this could be included in future work to evaluate the implications of delaying maintenance and repairs.

Once the stabilised number of defects in each group has been determined, the expected number of inspections, repairs and failures each year can be calculated by multiplying by the probability of inspection (for detection and sizing), the probability of repair and the probability of failure (between inspections and at an inspection year), respectively.

The cost of an individual inspection, which is directly proportional to the quality of the inspection, is calculated using Equation (15), for inspection 1, and Equation (16) and Equation (17) for inspection 2. The expected total cost of inspections is the product of the number of inspections and the cost of an individual inspection.

$$
\begin{gathered}
C I 1=C_{0} k_{I 1}\left(Q_{1} / Q_{\text {ref }}\right)^{0.5} \\
C I 2=C_{0} k_{I 2}\left(Q_{2} / Q_{\text {ref }}\right)^{0.5} \\
k_{I 2}=\eta k_{I 1}
\end{gathered}
$$

where $C_{o}=$ initial construction cost, $k_{I 1}=$ inspection cost coefficient for detection, $k_{I 2}=$ inspection cost coefficient for sizing, $Q_{1}=$ inspection quality for detection, $Q_{2}=$ inspection quality for sizing assessment, $Q_{\text {ref }}=$ reference inspection quality and $\eta=$ ratio of inspection cost coefficients for detection and sizing.

Using this non-linear cost model, for a specific increase in inspection quality $(\Delta Q)$, the cost increase for the inspection is less for higher values of inspection quality. For example, increasing $Q_{1}$ from 5 to 10 (thus reducing the standard deviation of the noise distribution by $50 \%$ ) results in a higher cost than increasing $Q_{1}$ from 45 to 50 (thus reducing the standard deviation of the noise distribution by $10 \%$ ). The reference inspection quality $\left(Q_{\text {ref }}\right)$ is used as a normalising factor. It is also assumed that the cost of the second inspection is less than the cost of the first inspection, due to the discounted cost of multiple inspections (e.g. costs of transporting equipment, setup costs, erecting scaffolding etc.). For this reason the cost coefficient for the sizing $\left(k_{i 2}\right)$ is assumed to be a fraction $(\eta)$ of the cost coefficient of the detection $\left(k_{i 1}\right)$.

Similarly, the cost of an individual repair (for each group) is calculated, Equation (18), and is then used to find the expected total cost of repair.

$$
C R=C_{0} k_{\mathrm{R}}
$$

where $k_{\mathrm{R}}=$ repair cost coefficient. It is assumed that the cost of repair is constant, depending only on the initial construction cost $\left(C_{o}\right)$ and the repair cost coefficient $\left(k_{\mathrm{R}}\right)$. In practice, the cost of repair may consist of a fixed and a variable component, where the variable component depends on the extent of the repair being carried out. However, the type of costing model would depend on the deterioration mechanism and defect type being considered, and various other costs associated with repair (e.g. labour costs, material costs, site setup costs, etc.). However, since this would be specific to the material deterioration mechanism, it is assumed for the purpose of this study that the repair cost is constant.

The cost of an individual failure at an inspection year is assumed to be equal to the cost of an individual failure between inspections. This cost is calculated using Equation (19). The cost of failure is assumed to be a multiple of the initial cost of construction $\left(C_{o}\right)$. The failure impact coefficient $\left(k_{\mathrm{F}}\right)$ is decided upon based on the impact of failure, which depends on factors such as the importance of the structure or structural component and the limit state being considered (e.g. SLS or Ultimate Limit State (ULS)).

$$
C F=C_{0} k_{\mathrm{F}} .
$$

Knowing the number of failures at the detection stage, the number of failures at the sizing assessment stage (at an inspection year) and the total number of failures between inspections, the expected total cost of failure is calculated.

The expected total cost of the structure is calculated by summing these costs. These costs are calculated over one $\Delta T$ cycle, therefore, the expected annual costs,

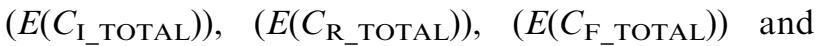
$\left(E\left(C_{\text {TOTAL }}\right)\right)$ are found by dividing by $\Delta \bar{T}$. 


\section{Results}

The possible range of defect sizes was subdivided into 10 groups (similar to the National Bridge Inventory Condition Rating system in the US). For the purpose of this example, the form of deterioration considered is cracking of concrete due to chloride-induced corrosion of the reinforcing bars. The defect size is assumed to vary from 0 to $1.0 \mathrm{~mm}$ since the limit crack width is considered to be $1.0 \mathrm{~mm}$ for this deterioration process (Vu 2003, Vu et al. 2005).

Table 1 presents the assumed set of parameters that were used as inputs to the model for the purpose of the results presented in this study. Since the objective of this work is the development of a maintenance management methodology, and no data are readily available for the parameters describing the deterioration, inspection, repair and failure of defects over time, a set of parameters is assumed to illustrate the capabilities of the methodology. Further work is required to calibrate the parameters used in this model to actual data.

Using these parameters presented in Table 1, the optimum time between inspections was determined on the basis of the minimum expected mean annual total cost of the structure, $\left(E\left(C_{\text {TOTAL }}\right)\right)$, which was assessed according to the cost functions outlined in the Section above. These cost functions were used to estimate the expected mean annual cost of inspections, repairs and failures for a network of structures. Using these cost

Table 1. Parameter values used in Markov maintenance example.

\begin{tabular}{lr}
\hline & $\begin{array}{c}\text { Assumed } \\
\text { Vodel properties }\end{array}$ \\
\hline Growth of defect & \\
Growth rate, $\alpha$ & 0.5 \\
Deterioration kinetics parameter, $g$ & 3 \\
Reference defect size, $d_{\text {ref }}$ & 1.0 \\
Probability of failure & 4 \\
Probability of failure exponent, $m$ & 0.3 \\
Limit defect size, $d_{1}$ & 1.0 \\
Reference defect size, $d_{\text {ref_pf }}$ & \\
Detection & 0.2 \\
Detection threshold, $d_{\text {min }}$ & 10 \\
Quality of inspection for detection, $Q_{1}$ & 0.1 \\
Mean of noise distribution, $n_{\text {mean }}$ & 0.62 \\
Sizing assessment & 20 \\
Critical defect size, $d_{c}$ & 1000 \\
Quality of inspection for sizing assessment, $Q_{2}$ & 0.01 \\
Cost analysis & 0.2 \\
Initial construction cost, $C_{o}$ & 20 \\
Inspection coefficient, $k_{I 1}$ & 0.1 \\
Ratio of inspection cost coefficients, $\eta$ & 1.0 \\
Reference quality, $Q_{\text {ref }}$ & \\
Repair coefficient, $k_{\mathrm{R}}$ & \\
Failure impact coefficient, $k_{\mathrm{F}}$ & \\
\hline
\end{tabular}

estimations it is possible to study the effect of the inspection interval on the expected mean annual costs. Based on this information, the owner/manager can decide on the optimal inspection interval, which in this case is based on the minimisation of the expected mean annual total cost. Figure 9 shows the results of the analysis, illustrating that for the case considered a period of 3 years represents the optimum inspection interval.

As illustrated in Figure 9, the inspection interval has a significant effect on the expected mean annual inspection cost, $\left(E\left(C_{\text {II_tOTAL}_{1}}\right)\right)$, and the expected mean annual failure cost, $\left(E\left(C_{\mathrm{F}_{-} \text {тоTAL }}\right)\right)$. The expected inspection cost ranges from $52 \%$ of the total cost for a 1 -year inspection interval to just $5 \%$ of the total cost for a 10-year inspection interval. As expected, an inverse trend emerges for the failure cost, with the expected failure cost ranging from just $4 \%$ of the total cost at a 1-year inspection interval to $77 \%$ of the total cost for a 10-year inspection interval. For example, considering a service life of 80 years, a 1-year inspection interval results in 80 inspections over the service life, resulting in a high inspection cost but a low failure cost since all defects are detected and subsequently repaired when they are found to be greater than the critical defect size. For an inspection interval of 10 years, however, there are only eight inspections over the service life, resulting in a lower annual inspection cost but a higher failure cost since defects are undetected and unrepaired for a period of 10 years between inspections, resulting in failures.

Figure 9 also illustrates the effect of the inspection interval on the expected cost of repair. As the inspection interval increases, there is a reduction in the number of repairs since inspections are carried out less often. For longer inspection intervals, defects are

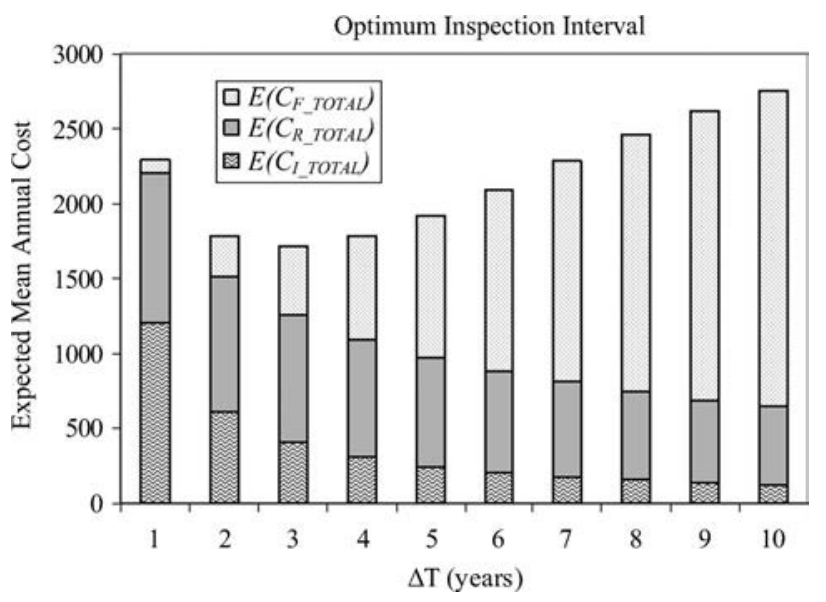

Figure 9. The effect of the inspection interval on expected mean annual costs. 
more likely to fail (and be repaired) between inspections, and therefore do not require repair after an inspection is carried out.

Using the developed methodology, it is also possible to look at the interaction of the quality of the inspection techniques for detection and sizing, and see how this affects the optimum inspection interval and the expected mean annual total cost. By varying the inspection quality of both techniques independently, the optimum combination of techniques can be found, which results in the minimum expected mean annual total cost. This provides the owner/manager of a structure with a useful decision tool when selecting a combination of inspection techniques to be used as part of a maintenance management plan, rather than using the same quality inspection technique for both stages of an inspection. Figure 10a-f illustrates for an inspection interval of 1-6 years, how a different combination of inspection techniques can affect the expected mean annual cost of the structure. An inspection interval of 1-6 years was studied, as principal inspections are recommended every 6 years in the UK (Vassie and Arya 2006), and from 1 to 6 years in Ireland, depending on the condition of the structure and the environment (Duffy 2004).

In relation to the first inspection, a higher quality technique, $Q_{1}$, reduces the noise associated with the inspection procedure (Equation (12)), and therefore, more accurately determines which defects should be further assessed, which consequently reduces the number of failures due to undetected defects. Similarly, in relation to the quality of the second technique, $Q_{2}$, a better technique reduces the number of failures, as a higher proportion of defects are sized correctly and repaired when necessary. For each inspection interval, by varying the inspection quality of both techniques
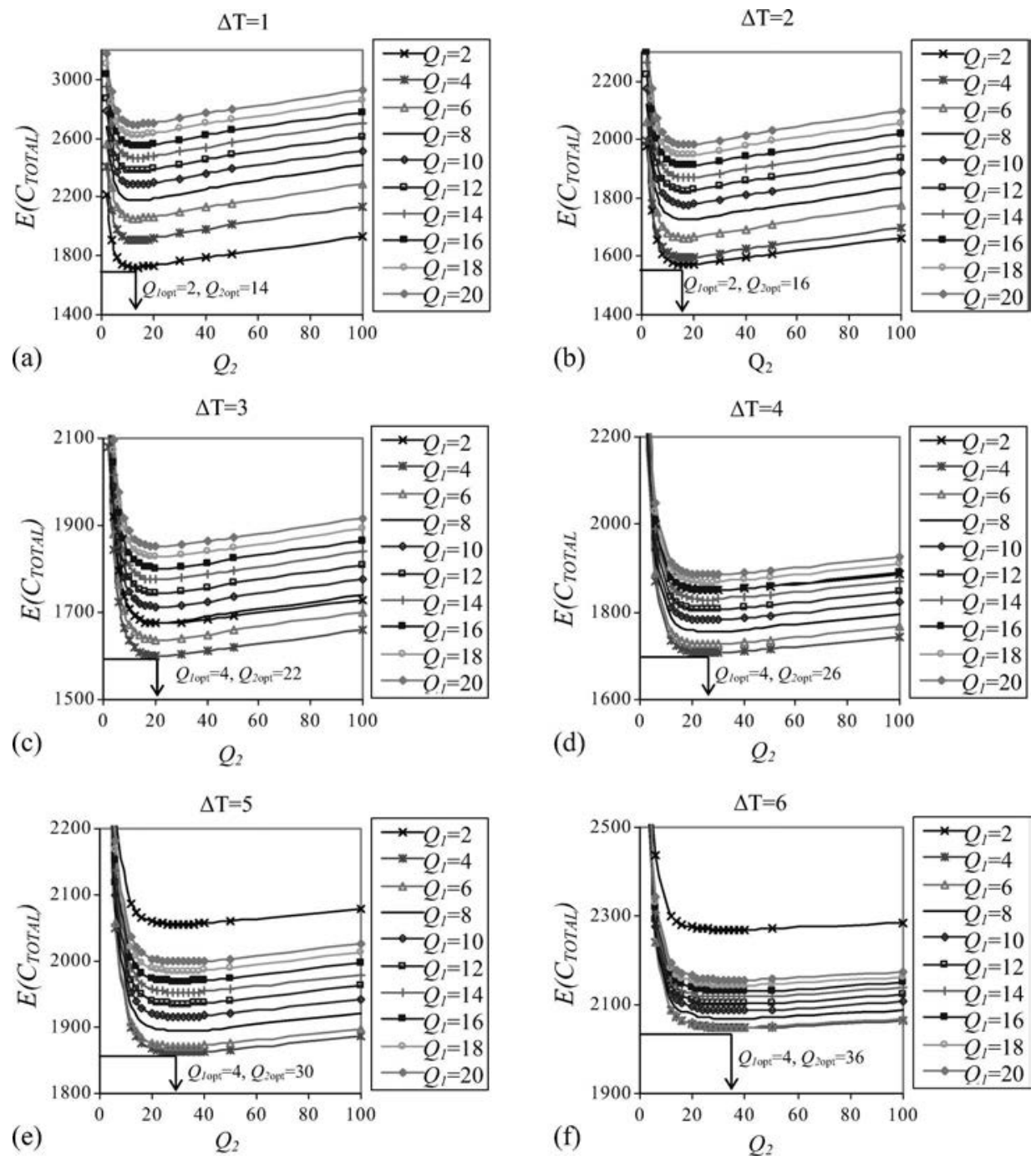

Figure 10. Effect of the inspection quality on the total cost (for $\Delta T=1: 6$ ). 
independently, the optimum combination of techniques and the corresponding expected mean annual total cost were found.

The results of the analysis are presented in Table 2 . Based on the minimum expected mean annual total cost, the optimum inspection interval was 2 years, as presented in Table 2. Also presented is the percentage increase in the expected cost for each inspection interval, with respect to the minimum total cost (i.e. @ $\Delta T=2$ ). This allows the owner/manager of a structure to see the effect of increasing the inspection interval when the optimum combination of inspection techniques is used to carry out the inspection for detection and sizing assessment.

For example, the expected increase in total cost by increasing the inspection interval from 2 (the optimum inspection interval) to 6 years is $30 \%$. The optimum quality of both inspections increases with inspection interval. The optimum combination of techniques, which results in the minimum expected mean annual total cost, are illustrated in Figure 10. The optimum inspection quality for detection is very low (with an associated high level of noise), varying from $Q_{1}=2$ to $Q_{1}=4$ for the limit state considered (i.e. SLS, corrosion induced cracking of reinforced concrete). This first inspection represents a screening exercise to determine which defects require further inspection. Inaccurate inspection results in a higher number of second inspections or a small possibility of failure.

The optimum inspection quality for sizing is much higher, ranging from $Q_{2}=14$ to $Q_{2}=36$, representing moderate to high quality inspection techniques, which are associated with a lower level of noise. These values are highlighted by arrows in Figure $10 \mathrm{a}-\mathrm{f}$, for an inspection interval ranging from 1 to 6 years. In relation to the second inspection, the consequence of inaccurate sizing is more serious than an inaccurate inspection for detection, as defects greater than $d_{c}$ that are not repaired are likely to result in failure (i.e. exceedance of the limit state). A better technique reduces the number of failures, as a higher proportion of defects are sized correctly and repaired when necessary.

Table 2. Optimum combination of inspection techniques for each inspection interval.

\begin{tabular}{ccccc}
\hline$\Delta T$ & $Q_{1}$ & $Q_{2}$ & $E\left(C_{\text {TOTAL }}\right)$ & $\begin{array}{c}\text { Increase with respect to } \\
\text { minimum cost }(\Delta T=2)\end{array}$ \\
\hline 1 & 2 & 14 & 1724 & $10 \%$ \\
2 & 2 & 16 & 1571 & $0 \%$ \\
3 & 4 & 22 & 1600 & $2 \%$ \\
4 & 4 & 26 & 1706 & $9 \%$ \\
5 & 4 & 30 & 1863 & $19 \%$ \\
6 & 4 & 36 & 2048 & $30 \%$ \\
\hline
\end{tabular}

This suggests that the manager of a structure should focus more resources on phase 2 (sizing assessment) of the inspection than phase 1 (detection), as indicated by the results of this analysis. When carrying out a traditional one-stage inspection, the detection threshold $\left(d_{\min }\right)$ is essentially the same as the repair threshold, as all defects that are detected are automatically repaired. This two-stage methodology allows the owner/manager of a structure to specify a detection threshold, so that defects that are present in the structure can firstly be detected, and also specifies a repair threshold, where defects sizes that are found to be greater than this threshold can be repaired.

\section{Conclusions}

This work has demonstrated the capabilities of the developed Markov maintenance model. Firstly, it was shown how the methodology makes operable the determination of the optimal inspection interval for a particular set of input parameters. The variation in the different costs, such as inspection cost, repair cost and failure cost was illustrated for a range of inspection intervals, which in this case resulted in an optimum inspection interval of 3 years. Using the developed framework, the optimal inspection interval for an infrastructural element/network can be assessed based on the minimisation of the service life costs for a specified limit state.

When carrying out an inspection there are two points of interest, the presence of a defect and the size of a defect present. Since each stage of the inspection has a different purpose, it is necessary to separate these procedures to accurately model an inspection process which is to be incorporated into a maintenance management plan. The separation of the inspection process of a structure into two stages enables the investigation to study the effect of both stages of the inspection on the expected annual costs of the structure, and the maintenance plan for the structure. Budgets are allocated to infrastructure managers each year for the implementation of inspection programmes. This developed methodology enables the optimisation of this process by the selection of specific methods at each stage of an inspection as a function of many different factors (such as environment, deterioration mechanism, severity of deterioration, etc.) to deliver maximum benefits. The separation of these procedures and the interaction of the two inspection techniques have not previously been considered. Depending on the requirements of the owner/manager, it may be more convenient to use a low-quality screening technique for detection and a higher quality inspection technique for sizing to assess which defects should be repaired. The developed methodology allows 
for the first time the effect of such decisions to be evaluated. In conclusion, using this methodology, the optimum combination of techniques can be determined for each stage of an inspection, to optimise budget spend.

\section{Acknowledgement}

The support of the European Union Interreg IIIb - Atlantic Area program through funding of the MEDACHS research project (Project No. 197) is gratefully acknowledged.

\section{References}

Adey, B., Bernard, O., and Gérard, B., 2003. Riskbased replacement strategies for deteriorating reinforced concrete pipes. In: Proceedings of 2 nd International RILEM Workshop on Life Prediction and Aging Management of Concrete Structures, Paris, France, RILEM, 373382.

Ang, A.H-S. and Tang, W.N., 1975. Probability concepts in engineering planning and design volume I: basic principles. New York: John Wiley and Sons.

Ang, A.H-S. and Tang, W.N., 1984. Probability concepts in engineering planning and design volume II: decision risk and reliability. New York: John Wiley and Sons.

Bakht, B. and Mutsuyoshi, H., 2005. Development of bridge management system (BMS) in Japan and USA. In: Proceedings of the 5th International Conference on Bridge Management, University of Surrey, UK, Thomas Telford Ltd, 37-43.

Cesare, M.A., et al., 1992. Modelling bridge deterioration with Markov chains. Journal of Transportation Engineering, 118 (6), 820-833.

Chung, H.Y., Manuel, L., and Frank, K.H., 2006. Optimal inspection scheduling of steel bridges using nondestructive testing techniques. ASCE Journal of Bridge Engineering, 11 (3), 305-319.

Corotis, R.B., Ellis, J.H., and Jiang, M., 2005. Modelling of risk-based inspection, maintenance and life-cycle cost with partially observable Markov decision processes. Structure and Infrastructure Engineering, 1 (1), 75-84.

Czepiel, E., 1995. Bridge management systems literature review and search. Northwestern University Infrastructure Technology Institute, Technical Report No. 11.

Duffy, L., 2004. Development of Eirspan: Ireland's bridge management system. Proceedings of the Institution of Civil Engineers: Bridge Engineering, 157 (3), 139146.

Enright, M.P. and Frangopol, D.M., 1999. Maintenance planning for deteriorating concrete bridges. Journal of Structural Engineering, 125 (12), 1407-1414.

Estes, A.C. and Frangopol, D.M., 1999. Repair optimization of highway bridges using system reliability approach. Journal of Structural Engineering, 125 (7), 766-775.

Estes, A.C. and Frangopol, D.M., 2001. Bridge lifetime system reliability under multiple limit states. Journal of Bridge Engineering, 6 (6), 523-528.

Faber, M.H. and Sorensen, J.D., 2002. Indicators for inspection and maintenance planning of concrete structures. Journal of Structural Safety, 24 (4), 377-396.

Frangopol, D.M., Kong, J.S., and Gharaibeh, E.S., 2001. Reliability-based life-cycle management of highway bridges. Journal of Computing in Civil Engineering, 12 (1), 27-34.
Kong, J.S. and Frangopol, D.M., 2004. Cost-reliability interaction in life-cycle cost optimization of deteriorating structures. Journal of Structural Engineering, 130 (11), 1704-1712.

Kong, J.S. and Frangopol, D.M., 2005. Probabilistic optimization of ageing structures considering maintenance and failure costs. Journal of Structural Engineering, 131 (4), 600-616.

Madsen, H., et al., 1987. Probabilistic fatigue crack growth analysis of offshore structures, with reliability updating through inspection. Marine Structure Reliability Symposium, 5-6 October 1987, Arlington, Virginia, $45-55$.

O'Connor, A. and Eichinger, E., 2007. Site-specific traffic load modelling for bridge assessment. ICE Journal of Bridge Engineering, 160 (4), 184-194.

O'Connor, A. and Enevoldsen, I., 2009. Probability based assessment of bridges according to the new Danish guideline. Structure and Infrastructure Engineering, 5 (2), 157-168,

O'Connor, A. and O'Brien, E., 2005. Mathematical traffic load modelling and factors influencing the accuracy of predicted extremes. Canadian Journal of Civil Engineering, 32 (1), 270-278.

Onoufriou, T. and Frangopol, D.M., 2002. Reliability-based inspection optimisation of complex structures: a brief retrospective. Journal of Computers and Structures, 80 (12), 1133-1144.

Radojicic, A., Bailey, S.F., and Brühwiler, E., 2001. Probabilistic models of cost for the management of existing structures. Life-Cycle Cost Analysis and Design of Civil Infrastructure Systems, SEI-ASCE. USA: ASCE, 251-270.

Rens, K.L., Nogueira, C.L., and Transue, D.J., 2005. Bridge management and non-destructive evaluation. Journal of Performance of Constructed Facilities, 19 (1), 3-16.

Roelfstra, G., et al., 2004. Condition evolution in bridge management systems and corrosion-induced deterioration. ASCE Journal of Bridge Engineering, 9 (3), 268-277.

Rouhan, A. and Schoefs, F., 2003. Probabilistic modeling of inspection results for offshore structures. Journal of Structural Safety, 25 (4), 379-399.

Scherer, W.T. and Glagola, D.M., 1994. Markovian models for bridge maintenance management. Journal of Transportation Engineering, 120 (1), 37-51.

Sheils, E., et al., 2010. Development of a two stage inspection process for the assessment of deteriorating infrastructure. Reliability Engineering and System Safety, submitted for publication.

Stewart, M.G., 2001. Reliability based assessment of ageing bridges using risk ranking and life cycle cost decision analyses. Reliability Engineering and System Safety, 74 (3), 263-273.

Stewart, M.G., Estes, A.C., and Frangopol, D.M., 2004. Bridge deck replacement for minimum expected cost under multiple reliability constraints. Journal of Structural Engineering, 130 (9), 1414-1419.

Straub, D. and Faber, M.H., 2003. Modeling dependency in inspection performance. In: A. Der Kiureghian, S. Madanat, and J. Pestana, eds. 9th International Conference on Applications of Statistics and Probability in Civil Engineering, 6-9 July 2003, San Francisco. Millpress, $1123-1130$.

Vassie, P.R. and Arya, C., 2006. Long-term maintenance strategies for highway bridges. Proceedings of the Institution of Civil Engineers: Bridge Engineering, 159 (2), 83-90. 
Vu, K.A.T., 2003. Corrosion induced cracking and spatial time dependent reliability analysis of reinforced concrete structures. Thesis (PhD). University of Newcastle, Newcastle, Australia.

Vu, K.A.T., Stewart, M.G., and Mullard, J.A., 2005. Corrosion-induced cracking: experimental data and predictive models. ACI Structural Journal, 102 (5), 719-726.
Weibull, W., 1951. A statistical distribution function of wide applicability. Journal of Applied Mechanics, 18 (3), 293-297.

Zhang, R. and Mahadevan, S., 2001. Fatigue reliability using nondestructive inspection. Journal of Structural Engineering, 127 (8), 957-965. 